\title{
Strukturell-funktionale Theorie und Psychoanalyse. Die Pionierarbeit von Talcott Parsons
}

\author{
Johann August Schülein
}

Zusammenfassung: Zu den bleibenden Leistungen von Parsons gehört u.a. auch, dass er in seinen strukturell-funktionalen Entwurf einer Gesellschaftstheorie einige der theoretischen Leistungen der Psychoanalyse eingebaut hat. Mit Hilfe der Konzepte internalisierter Normativität („Über-Ich“), intrapsychischer Kontrolle und Steuerung und psychisch organisierter Objektbeziehungen gelingt es ihm, die Funktionsweise von Goal-Attainment, aber auch von Integration und Latency differenzierter darzustellen. Zugleich trägt seine kritische Auseinandersetzung dazu bei, die Schwächen von Freuds Theorien in Bezug auf den Austausch von Psychischem und Sozialem zu überwinden und dadurch den Weg in Richtung auf eine bessere Kooperation von Soziologie und Psychoanalyse zu bahnen.

Schlüsselwörter: Strukturell-funktionale Theorie, Psychoanalyse

\section{Structural-functional theory and psychoanalysis. Talcott Parsons' groundbreaking work}

\begin{abstract}
Parsons' attempts to integrate psychoanalytic concepts into sociological theory belong to the highlights and remaining achievements of his work. Using the psychoanalytic ideas of the internalization of norms and goals ("super-ego"), of intrapsychic regulation and of psychic organized objectrelations Parsons gained deeper insights in the functioning of Goal-Attainment, Integration and Latency and by this a more complete picture of social cohesion. On the other hand, Parsons' critical discussion of Freud's
\end{abstract}

Johann August Schülein $\square$

WU - Wirtschaftsuniversität Wien, Institut für Soziologie und Empirische Sozialforschung

Welthandelsplatz 1, Gebäude D4, 1020 Wien, Österreich

E-Mail: johann.schuelein@wu.ac.at 
theories helped to overcome their faibles and thereby opened the path to a better cooperation of sociology and psychoanalysis.

Keywords: Structural-functional theory, psychoanalysis

Der Name Parsons steht für ein ebenso ambitioniertes wie prominentes Projekt soziologischer Theoriebildung: die Strukturell-funktionale Theorie. Diese Theorie war für lange Zeit die Leittheorie zumindest der angelsächsischen Soziologie, sie wird auch heute noch zu den wichtigsten soziologischen Theoriemodellen gezählt.

In Parsons' Konzeption spielte die Psychoanalyse eine wichtige Rolle. Er sah in der psychoanalytischen Theorie einen geradezu natürlichen Verbündeten seines ehrgeizigen Projekts, weil sie aus seiner Sicht strukturelle Ähnlichkeiten mit seinem Verständnis soziologischer Theorie aufweist, andererseits die Soziologie in Schlüsselfragen ergänzt. - Naturgemäß nahm die Ausarbeitung seines Projekts einige Zeit in Anspruch. Zunächst versuchte Parsons, die vorhandenen Paradigmen zu ordnen und in Verbindung zu bringen. In der ersten Phase seiner Arbeit versuchte er vor allem, dass „utilitaristische Dilemma“ zu überwinden. Unter dem Namen „Utilitarismus“ hatte sich im 18. und 19. Jahrhundert ein vor allem in ökonomischen Theorien verwendetes Konzept entwickelt, welches als Grundprinzip allen menschlichen Handelns die „Nützlichkeit“ annahm. Als zugespitzte Form entstand dabei das Modell des „homo oeconomicus“, der in jeder Handlung seinen Nutzen nach einer festliegenden Präferenzordnung „maximiert“ (welches sich auf Grund dieser Eindeutigkeit zur Quantifizierung eignet und daher in quantitativen Modellen nach wie vor Verwendung findet).

Das „utilitaristische Dilemma“ bestand nach Parsons darin, dass der Utilitarismus annehmen muss, dass die Ziele der Handlungen - also das, was als „nützlich“ unterstellt wird - als völlig gleich bzw. austauschbar sind (eine Beliebigkeit, die nach Parsons zu einer Art von atomistischer Anarchie der sozialen Wirklichkeit führen würde und daher keine Grundlage der Soziologie sein kann). Wenn nicht, müsste er jedoch externe Prämissen heranziehen, um die Herkunft der Nutzenvorstellungen zu erklären (was nach Parsons die ,analytische Selbständigkeit“ der Theorie auflöst - wozu braucht man den Utilitarismus noch, wenn das Wichtigste aus anderen Theorien stammt?). Um aus diesem Dilemma - entweder Aufgabe des Sinns von Handlungen durch Ausklammerung oder Aufgabe der analytischen Selbständigkeit der Theorie - zu vermeiden, entwickelte Parsons unter Berufung auf eine Reihe von wichtigen Vertretern der Ökonomie und Soziologie eine erste Konzeption, die sich allem auf Alfred Marshall (der die Rolle gemeinsamer Wertschätzungen hervorhob), Vilfredo Pareto (und seine Betonung nicht-rationalen Verhaltens), Emile Durkheim (der ein Konzept von Kollektivbewusstsein als Bindeglied von 
Individuum und Gesellschaft entwickelte) und Max Weber (der die Intentionalität von Handlungen zum Ausgangspunkt nahm) berief.

Heraus kam dabei ein erstes Modell, in dem individuelle Handlungsmotive über die Internalisierung von Normen mit der Logik der Gesellschaft verbunden wurden. - In diesem Modell kommt die Psychoanalyse nicht systematisch vor. ${ }^{1}$ Zum Zeitpunkt der Veröffentlichung der „Structure of Social Action“ hatte Parsons jedoch bereits begonnen, sich intensiv mit psychoanalytischer Theorie zu beschäftigen - u. a. unterzog er sich einer Lehranalyse. Im Vorwort zur zweiten Auflage bedauerte Parsons, dass der Text nicht psychologisch genug sei und vor allem die Leistungen der Psychoanalyse nicht berücksichtigt habe. - Die Rezeption der Psychoanalyse vollzieht sich bei Parsons im Rahmen der Weiterentwicklung seiner Theorie. Dubin (1960, S. 462f.) beschreibt diesen Wandel so: „Model I essentially ,looks out' to the social system from the vantage point of the actor, his model II ,looks down" at the individual actor from the perspective of the social system." Insofern sprechen manche Kritiker von einem Übergang von der Handlungs- zur Systemtheorie. Tatsächlich erweitert sich Parsons' Perspektive erheblich. Auch der Anspruch an die Theorie steigt. Jetzt geht es ihm um eine allgemeine Theorie als Grundlage jeder Sozialwissenschaft (,general theory of action“) und jeder Form sozialer Praxis - ,[...] a unified conceptual scheme which is taken for granted in the relevant professions [...]" (Parsons 1964, S. 360)².

Parsons geht dabei davon aus, dass alle Systeme bestimmte Probleme und Eigenschaften haben, die mit ihrem Funktionieren zusammenhängen. Jedes System muss in seiner Umwelt funktionsfähig bleiben, muss Entscheidungen treffen, die auf Ziele bezogen sind, bedarf einer Form der Integration seiner Bestandteile und muss seine Struktur erhalten. Humane Wirklichkeit ist ein Sonderfall von System. Sie gehört zur Gruppe der Handlungssysteme, basiert also darauf, dass es Akteure gibt. Auch für diesen Sonderfall gilt nun nach Parsons, dass sie diese vier Grundfunktionen erfüllen müssen, um operationsfähig zu sein. Daraus entstand - als Anker der weiteren Entwicklung seiner Theorie - das so genannte AGIL-Schema. Es bezeichnet die vier Grundfunktionen jedes Handlungssystems, für das jeweils ein bestimmtes Subsystem zuständig ist:

- A: Adoption (Anpassung an die Systemumwelt und Generierung von Ressourcen - Parsons nennt diesen Systemtyp „behavioral system“)

- G: Goal-Attainment (Entscheidung über Ziele und deren Erreichung - zuständig ist dafür ein „Persönlichkeitssystem“)

1 Parsons verweist nur kurz auf die psychoanalytische Theorie der Internalisierung (1937, S. 386 und 388).

2 Die folgende Darstellung ist vereinfacht, selektiv und zum Teil anders konfiguriert als in den Originaltexten, da eine genaue Darstellung der Entwicklung von Parsons' Theorie diesen Rahmen sprengen würde. 
- I: Integration (Herstellung der Einheit des Systems und Kontrolle von internen Abweichungen - dies ist die Aufgabe eines sozialen Systems)

- L: Latency (Gewährleistung von Stabilität und Kontinuität des Systems durch „pattern maintainance").

Für jede dieser Grundfunktionen bildet sich also ein darauf spezialisiertes und dafür zuständiges Subsystem, welches nach eigenen Regeln operiert. Da diese Grundfunktionen für alle Handlungssysteme kennzeichnend sind, lässt sich auf diese Weise auch jedes soziale System - von konkreten Interaktionen über Familien und Organisationen bis zu ganzen Gesellschaften dadurch charakterisieren, wie diese bestandswichtigen Funktionen erfüllt werden.

Mit den Grundfunktionen und dem Prinzip der strukturierten Systembildung auf allen Ebenen hat Parsons seiner Theorie eine Basis gegeben. Eine wichtige Ergänzung zu dieser „Pyramide“ aus Systemen und Subsystemen war die Theorie der „pattern variables“, in der Parsons beschrieb, wie die Erfüllung der jeweiligen Funktionen variieren kann. Dabei unterschied er fünf Dimensionen von Funktionen, in denen sie in der Art der Funktionserfüllung zwischen zwei Polen liegen - also strukturell variieren - können:

- Affektivität - affektive Neutralität (werden die Funktionen sachlich nüchtern oder mit emotionaler Aufladung erfüllt?)

- Spezifizität - Diffusion (sind die Funktionen klar getrennt und fokussiert oder sind sie unscharf und vermischt?)

- Universalismus - Partikularität (sind die Funktionen in einem „Generalplan“ aufeinander bezogen oder werden sie getrennt voneinander behandelt?)

- Zugewiesen - erworben (haben die Akteure/Subsysteme die Funktionen zugewiesen bekommen oder haben sie sie sich selbst angeeignet und erworben?)

- Selbstorientierung - Kollektivorientierung (orientiert sich die Funktionserfüllung am Akteur/Subsystem selbst oder am Kontext, am Interesse aller Akteure bzw. des Gesamtsystems?).

Parsons` Vorstellung von sozialer Realität läuft also im Kern darauf hinaus, dass er sie als ein System betrachtet, welches prinzipiell bestimmte Grundfunktionen erfüllen muss, die in entsprechenden Substrukturen mit einem systemspezifischen Profil bewältigt werden und untereinander verbunden sein müssen. Daraus ergibt sich eine Pyramide von der Gesamtgesellschaft bis zu konkreten Situationen, die nach einem ähnlichen Schema operieren.

Für Parsons ist klar, dass auf jeder Systemebene spezifische Leistungen der Akteure erforderlich sind, damit das System funktionsfähig wird und bleibt. „Goal-attainment“, „Integration“ und auch „Latency“ erfordern spezifische psychische Leistungen. Es stellt sich daher die Frage, wie denn Gesellschaften und ihre Subsysteme die für sie erforderlichen Leistungen der Subjekte 
erzeugen und auswählen - ganz allgemein formuliert: wie ein dem sozialen System entsprechendes Persönlichkeitssystem zustande kommt.

Für einen engagierten Theoretiker muss allerdings vorher einiges geklärt werden. Wie muss eine Persönlichkeitstheorie aussehen, die zur eigenen Theorie passt? Und wie werden Soziologie und Persönlichkeitstheorie aufeinander bezogen? - Auf die erste Frage findet Parsons eine klare Antwort: Er zieht die Psychoanalyse dem Behaviorismus und dem Utilitarismus vor, weil sie Handeln unter dem Vorzeichen individueller Motivation betrachtet, ohne sie auf Reiz-Reaktionsmuster oder einen bestimmten Typus von Rationalität zu reduzieren (was die soziologische Verwendbarkeit einschränkt).

„Die Psychoanalyse ist meines Erachtens die erste und bis jetzt einzige theoretische Analyse der menschlichen Persönlichkeit, die als ein System von vergleichbarem Niveau unmittelbar mit der soziologischen Theorie vom Wesen und der Funktionsweise geordneter Modelle innerhalb des Systems menschlicher sozialer Beziehungen in Verbindung gebracht werden kann.“ (Parsons 1972, S. 96)

Das hängt nicht zuletzt damit zusammen, dass Freud gewissermaßen eine Systemtheorie der Persönlichkeit vorgelegt hat oder zumindest eine Theorie, die mit Parsons ${ }^{6}$ Modell kompatibel ist: Parsons stellt fest, dass Freuds Subjekttheorie genau so angelegt ist, dass sie dem entsprecht, was eine für seine Konzeption passende Theorie leisten muss:

„Freud's structural theory was certainly fundamentally on the right track in that it clearly formulated the three major points of reference of personality theory - the needs of the organism, the external situation, and the patterns of the culture." (Parsons 1953, S. 26)

Das passt gut zum AGIL-Schema. Die Frage nach dem gemeinsamen Arbeitsprogramm diskutiert Parsons im Aufsatz „Psychoanalysis and the Social Structure“ (1950). Er betont die logische und empirische Gleichrangigkeit von sozialem System und Persönlichkeitssystem, aber auch ihre Differenz, was zur Folge hat, dass die jeweiligen theoretischen Perspektiven - Soziologie und Psychologie - unterschiedlich operieren. Sie können sich nicht substituieren. Daher sind beispielsweise die Annahmen, die die Soziologie als Subjekttheorie nutzt, typischerweise zu vage ${ }^{3}$, um theoretisch produktiv zu sein. - Andererseits impliziert die Theoriedifferenz, dass die jeweils andere Sichtweise nicht einfach übernommen werden kann, sondern akkomodiert

3 „The motivational constructs needed for the solution of any sociological problem will generally turn out to be inadequate to explain the action of any particular individual [...]“ (1950, S. 341), so dass Soziologen häufig zu ad-hocErklärungen oder empirischen Generalisierungen greifen. Umgekehrt verwenden Psychologen für ihre Erklärungen individuellen Verhaltens ad-hoc-Erklärungen der konkreten sozialen Bedingungen, die theoretisch ungenügend seien (a. a. O.: 341f.). 
werden muss, um zu passen. Das Ziel ist daher keine „psychologische Soziologie“, sondern eine Soziologie, die psychologische Erkenntnisse in ihrem Kontext verwendet.

Im Lauf der Arbeit an seinem Gesamtkonzept hatte Freud eine ganze Reihe von Themen behandelt, die in seiner Sicht eine Kooperation von Soziologie und Psychoanalyse sinnvoll erscheinen ließen. Dazu gehörten vor allem folgende Punkte:

- Bei der Formulierung der „pattern variables“ hatte er sich ausdrücklich auf Freud bezogen und die Unterscheidung zwischen Affektivität und affektive Neutralität mit dessen psychischen Instanzen „Es“ und „Ich“ ebenso Selbst- und Kollektivorientierung mit Ich und Über-Ich (Parsons et al. 1956, S. 85) in Verbindung gebracht. Dazu postuliert Parsons das Ich als Doppelfunktion und unterscheidet zwischen dem adaptivem Ich, welches affektive Neutralität braucht und gewährleistet, und dem integrativen Ich, welches die Selbstorientierung trägt und entsprechend erlebt und steuert (d.h. auch: triebhaft agiert). ${ }^{4}$

- Grundlegend für das Funktionieren von sozialen Systemen ist für Parsons die Frage, wie Akteure überhaupt dazu kommen, sich an gesellschaftlichen Vorgaben zu orientieren. Dazu muss die Frage der , , [...] balance of motivational forces involved in the maintainance of, and alteration in, the structure of a social system [... ]" (Parsons 1950, S. 340) geklärt werden. In diesem Zusammenhang sieht er eine wichtige Funktion der Psychoanalyse in ihrem Beitrag zur Erklärung der Genese und Formatierung psychischer Energie für das soziale System, wobei er das Konzept der Objektbesetzung (cathexis) von Freud übernimmt.

- Zugleich bietet die Psychoanalyse auch eine „dynamic theory of motivation“ (Parsons 1950, S. 339), die es erlaubt, die umgekehrte Frage zu klären: Wie kommt die Gesellschaft ins Individuum? Parsons hat in diesem Zusammenhang die Schlüsselinstanz der Verbindung von Psyche und Gesellschaft vor allem im Freud'schen Über-Ich gesehen (Parsons 1953).

Im weiteren Verlauf seiner Arbeit erweiterte und systematisierte Parsons seine Perspektive. Im Aufsatz „Sozialstruktur und Persönlichkeitsentwicklung: Freuds Beitrag zur Integration von Psychologie und Soziologie“ (1958a) beschäftigt er sich ausführlich mit der Art und Weise, wie die verschiedenen Verknüpfungspunkte integriert werden können.

Der Ausgangspunkt auch hier: Soziale System sind auf spezifische Leistungen der Psyche angewiesen, die ihrerseits auf das Vorhandensein eines sozialen Kontextes angewiesen ist, weil

4 Zudem schlägt er vor, das Ich als Doppelsystem zu sehen, weil es zwei verschiedene Ursprünge hat und daher zwei verschiedene Anpassungsleistungen vertritt: organismische Adaption und psychisches Realitätsprinzip. „It would be derived from two fundamental sources: the external world as an environment; and the common culture which is acquired from objects of identification“ (Parsons et al. 1956: 26). Zumindest in seiner Funktion als Anpassung an die soziale Umwelt ist das Ich für ihn daher ein Kulturprodukt. 
sonst sich weder entwickeln kann noch funktionsfähig ist. Um diesen Punkt zu verdeutlichen schlägt er eine Weiterentwicklung von Freuds Sichtweise vor:

„Freud's view was to narrow. [...] Not only moral standards, but all the components of the common culture are internalized as part of the personality structure. [...] The content of both cathetic-attitudes and cognitive-status definitions have cultural, hence normative significance. $[\ldots]$ Neither what the human object is, $[\ldots]$ nor what is means emotionally, can be understood independently of the nature of the interactive process itself." (Parsons 1953, S. 18f.)

Es gibt also keine Psyche, die a priori existiert und dann in Kontakt mit ihrer Umwelt kommt - sie ist von Anfang an Teil eines Gesamtprozesses, in dem sich Psyche und Sozialstruktur wechselseitig beeinflussen.

Parsons sieht dies jedoch als Tendenz, die im Freud'schen Konzept bereits angelegt ist, aber nicht immer deutlich zur Geltung kommt - Freud ist für ihn theoretisch gewissermaßen weiter als er selbst realisiert. Er verdeutlicht dies mit einer Rekonstruktion des Verhältnisses von Sozialisation - so nennt die Soziologie den Prozess der Anpassung von Akteuren an soziale Standards - und psychischer Entwicklung. Freud habe, so Parsons, mit den Begriffen Identifizierung, Objektbesetzung und Verinnerlichung/Introjektion eine Entwicklungslinie skizziert, die direkt die Entwicklung der sozialen Kompetenzen und Anforderungen beschreibt ${ }^{5}$.

Jeder der Begriffe steht für eine bestimmte Phase der Entwicklung. Gemeinsam ist ihnen, dass sie für eine prinzipielle Verbindung von Entwicklung und sozialem Kontext stehen. Deshalb sei es falsch, den gesellschaftlichen Einfluss auf das Über-Ich zu begrenzen: „Man hat versucht, das Über-Ich als einen Sonderfall innerhalb der Persönlichkeit zu behandeln, als die einzige Stelle, wo die Normen der Kultur in die Persönlichkeit eingehen." Tatsächlich gilt aber, „[...] daß dasselbe auch für die Struktur des Ichs gilt.“ (Parsons 2002, S. 101) Auch die „[...] Deutung des Es als Manifestation des, reinen Triebes' im Rahmen von Freuds eigenen Begriffen [...]“ ist für Parsons unhaltbar, denn ,[...] es ist ebenfalls durch verinnerlichte Objektbeziehungen [...]" (a.a.O.) strukturiert. Für ihn handelt es sich von Anfang an um eine Durchdringung („Interpenetration“) der vier analytisch unabhängigen, aber empirisch verbundenen Subsysteme Organismus, Persönlichkeit, soziales System und kulturelles System (aus denen ein Handlungssystem - entsprechend der vier Grundfunktionen - komponiert ist).

Identifizierung für Parsons der Begriff, der die frühe Mutter-Kind-Beziehung charakterisiert, eine Beziehung, die schon auf einer , ,...] sehr elementaren Ebene [...] einen echten Pro-

5 In diesem Zusammenhang verweist er darauf, dass es bemerkenswerte Parallelen zwischen Freud und vergleichbaren Bemühungen von Soziologen wie Durkheim, Cooley oder G.H.Mead gäbe - in seiner Sicht eine aussagekräftige Konvergenz. 
zeß sozialer Interaktion [...]“ (a.a.O., S. 107) darstellt, in dem sich das Motivationssystem des Säuglings auf das Muster der mütterlichen Aktionen inklusiver der damit verbundenen Ordnung einstellt. Dadurch entwickelt sich im Säugling ein

„System innerer Kontrollen über das Trieb- oder Impulssystem des Kindes durch ein von der Mutter auferlegtes, verallgemeinertes Regelmuster von Sanktionen - eine Form der Organisation des Ichs im Hinblick auf seine Beziehungen zu einem sozialen Objekt.“ (a.a.O., S. 110)

In der Phase der Objektbeziehung beginnt das Kind, ,[...] auf seine Objekte gerichtete Ziele aufzustellen. Hier wird die Objektwelt nicht einfach als gegeben behandelt. [...] Damit verschiebt sich das Schwergewicht seiner Rolle von Askription auf Leistung." (a.a.O., S. 117) Dem entspricht die Übernahme einer Mitgliedsrolle im familiären Milieu. - Die ödipale Phase markiert für die Entwicklung des Kindes aus soziologischer Sicht die Beziehung zur Familie als Gesamtsystem und zugleich die Differenzierung des familiären Beziehungsfeldes. Auf der Basis der Auseinandersetzung mit der Familie als differenziertem Objekt ist das „Über-Ich [...] primär das übergeordnete normative Muster, welches das Verhalten der verschiedenen Mitglieder in ihren verschiedenen Rollen innerhalb der Familie beherrscht [...]“ (aaO, S. 384). Die Über-IchBildung impliziert also ein höheres Maß an sozialer Komplexität und eine neues Niveau der Integration in den sozialen Prozess.

Parsons verbindet in dieser Interpretation die Beiträge der Psychoanalyse zur soziologischen Theoriebildung in einem entwicklungspsychologischen Konzept, in dem sich die soziale Reichweite des Handelns zugleich mit der Komplexität der verinnerlichten sozialen Realität erweitert. Dabei beruft er sich (gegen eine einseitig triebtheoretische Interpretation von Freud) auf Freuds Formulierung von 1923, das Ich sei „[...] der Niederschlag der aufgegebenen Objektbesetzungen [...]“ (Freud GW XIII, S. 257) und erweitert sie auch auf das Es, welches sich in seiner Sicht immer schon mit und in sozialen Formen entwickelt. Daher sind alle drei psychische Instanzen der Psyche und alle drei Stufen der Auseinandersetzung mit der Umwelt - Internalisierung, Objektbesetzung, Über-Ich-Bildung - von Anfang an gesellschaftlich bestimmt. Am Anfang steht die normativ bestimmte Symbiose mit der Mutter, am Ende das das kompetente Mitglied des Familiensystems mit Eigenkontrolle und -steuerung.

Parsons hat auf diese Weise seinen gesellschaftstheoretischen Entwurf an zentralen Punkten ausgebaut. Die persönlichkeits- und entwicklungstheoretischen Konzepte der Psychoanalyse erlauben es ihm, zugleich die Unabhängigkeit des „Persönlichkeitssystems“ vom „sozialen System" und deren wechselseitige Durchdringung zu erklären. Sie erlauben es ihm zudem - in der von ihm erweiterten Form - präzise zu bestimmen, über welche Mechanismen und Phasen sich Sozialisation und Enkulturation vollziehen. 
Parsons hat jedoch nicht nur ein abstraktes Modell entworfen, er hat seine Überlegungen immer wieder auf spezifische Themen angewandt. Ein Beispiel dafür ist die Arbeit über ,[...] Definitionen von Gesundheit und Krankheit im Lichte der amerikanischen Werte und Sozialstruktur Amerikas [...]“ (Parsons 1958b). Sie beschäftigt sich mit der Art und Weise, wie die Themen „Gesundheit“ und „Krankheit“ in unterschiedlichen Kulturen behandelt werden. Dazu greift er die Idee der Rolle als Verbindungsglied zwischen Individuum und Gesellschaft auf und erweitert sie durch die Unterscheidung zwischen „Rolle“ und „Aufgabe“ - der sozialen Erwartung und der Fähigkeit zur instrumentellen Auseinandersetzung mit der physischen Welt. Generell gilt für Gesundheit und Krankheit, dass sie bestimmt sind durch ihren Zusammenhang mit der individuellen Leistungsfähigkeit in diesem doppelten Sinn verbunden sind - „gesund“ ist, wer Rollenerwartungen und Aufgaben erfüllen kann.

So gesehen sind somatische Erkrankungen Störungen der instrumentellen Fähigkeiten, während psychische Erkrankungen verbunden sind mit der Unfähigkeit, sozialen Erwartungen zu entsprechen. Beides sind Störungen des sozialen Betriebs. Das Gesundheitssystem ist daher aufs Engste verbunden mit der Kontrolle und der Wiederherstellung von individueller Leistungsfähigkeit. Kranke wie Therapeuten stehen damit unter kulturell institutionalisiertem Rollendruck. Wer krank ist, darf zwar mit damit rechnen, einen Sonderstatus zu bekommen und von Aufgaben entlastet zu werden, muss aber seinerseits anerkennen, dass die Krankheit als unerwünscht gilt und ist verpflichtet, Hilfe zu suchen und zu nutzen, um diese Form der Abweichung von Normalität zu beseitigen oder zu reduzieren. Therapeuten sind die mit der Wiederherstellung von Normalität beauftragten Agenten des sozialen Systems.

Diese allgemeine Matrix wird kulturspezifisch variiert. Parsons führt vor, dass die amerikanische Gesellschaft mit ihrer Betonung von dem, was er „Aktivismus“, „Weltlichkeit“ und „Instrumentalismus“ nennt - also einem besonderen Profil der ,pattern variables“ in Richtung auf individuelle Zuständigkeit und arbeitsteilig-praktischer Bearbeitung - dazu tendiert, die Verantwortung des Einzelnen nicht für seine Krankheit, aber für deren aktive Bewältigung besonders hervorhebt. Der Kranke bekommt also leicht einen Sonderstatus - aber mit der Verpflichtung, ihn sobald wie möglich wieder zu verlassen ${ }^{6}$.

An dieser Stelle wird deutlich, warum psychische Krankheiten eine besondere Belastung für das soziale System und für seine Akteure darstellen ${ }^{7}$. Das zentrale Problem ist die Unfähigkeit zur Rollenerfüllung. Dafür gibt es - anders als bei Schwierigkeiten der Erfüllung von Aufgaben

6 Parsons kontrastiert das us-amerikanische Modell mit den - inzwischen verblichenen - sowjetischen und britischen Modellen, man könnte sagen: mit einem autoritär leistungsbetonten und einem autoritativ-apostolischen Modell.

7 Parsons diskutiert auch das Thema der Genese von psychischen Erkrankungen, wobei er aus der Freud'schen Konzeption die These ableitet, dass sie immer mit Beziehungsstörungen des familiären Milieus, also mit Problemen der Identifizierung, Objektbeziehungen und der Über-Ich-Bildung zumindest insofern zusammen hängt, als die Art des Umgangs mit Problemen dadurch bestimmt wird. Er ergänzt diese Feststellung mit dem Hinweis, dass hinter gestörten familiären Milieus gesellschaftliche Beziehungsstörungen stehen. - Das ändert nichts an der Verpflichtung zur (Wieder-)Herstellung von Leistungsfähigkeit. 
- keine soziale Exkulpation. Dies erhöht den Druck in Richtung auf Verleugnung, auf Umdefinition - oder in Richtung auf Korrekturmaßnahmen. Die Entstehung von Psychotherapie als Institution ist, so gesehen, eine Ausweitung und Modernisierung des sozialen Kontrollsystems. Parsons spricht davon, dass es darum geht, lizensierte Abhängigkeit zu nutzen, um Unabhängigkeit zu gewinnen - das schließt die psychoanalytische Nutzung von Übertragungsbeziehungen ein. Dahinter steht letztlich die kulturell verankerte Aufforderung zur Wiederherstellung der Leistungsfähigkeit. - Dass sich die Akzeptanz von Psychotherapie vor allem in den USA durchgesetzt hat, erklärt Parsons u.a. mit dem strukturellen Auftrag, vorhandene Chancen ,[...] zur konstruktiven therapeutischen Arbeit [...]“ (aaO, S. 363) zu nutzen.

Mit dieser Anwendung auf das Thema Krankheit/Gesundheit hat Parsons sein Modell einerseits konkretisiert und gezeigt, dass sein systemtheoretisches Konzept Themen sinnvoll formatieren kann. Andererseits kann er das Thema so aufbereiten, dass seine Mechanismen und sein sozialer Status deutlich werden. Dabei ist die psychoanalytische Theorie ein wichtiges Modul im Gesamtkonzept. Auffällig sind die Akzentverschiebungen, die Parsons für die Verbindung von Soziologie und Psychoanalyse vornimmt. Er folgt Freud in der Strukturtheorie, betont jedoch wesentlich stärker als Freud die genuine Beziehungs-(und damit Kultur-)Imprägniertheit aller psychischer Instanzen wie auch des akuten psychischen Funktionierens. Freud habe diese Gemeinsamkeit zu wenig gesehen. Seine Konzeption

„[...] tends to dissociate the superego from the sources of affect. This derives from the fact that Freud apparently did not appreciate the presence and significance of a common culture of expressive-affective symbolism and the consequent necessity for thinking of the emotional component of interaction as mediated by this aspect of common culture." (Parsons 1953, S. 20)

Dies gilt auch für das Es:

„Emotions, or affect on the normal human adult level, should be regarded as the symbolically generalized system, that it is never, id-impulse' as such. Affect is not a direct expression of drive-motivation, but involves it only as it is organized and integrated with both the reality experience of the individual and the cultural patterns which he has learned through the processes of identification." (a.a.O.)

8 Während in der UdSSR beispielsweise die Psychoanalyse als Bedrohung erlebt wurde, weil sie die Autonomie der Akteure (und von Therapie!) zu sehr betont und in Großbritannien der Druck zur (Selbst-)Korrektur nicht sonderlich ausgeprägt war, konnte die Psychoanalyse in den USA daher auch Karriere machen - allerdings als IchPsychologie, die gegen den alteuropäischen Skeptizismus die Möglichkeit zur (Rückgewinnung von) Autonomie hypostasiert. 
Affektive Objektbesetzungen, aber auch Komponenten der Identität sind daher die biografisch modifizierte Rückkehr gesellschaftlicher Muster.

Trieb und Lustprinzip werden von Parsons als wichtige Referenzen gesehen, aber wenig ausgearbeitet. Damit steht er interaktionstheoretischen Konzeptionen der Psychoanalyse nahe 9 - Unabhängig davon, ob man seinen Rekonstruktionen psychoanalytischer Theorie folgen will oder nicht: Sie stellen in jedem Fall ein Konzept dar, welches es ermöglicht, Freuds „,kulturtheoretische" Ambitionen auf einem wesentlich höherem Niveau in Angriff zu nehmen. Dazu bietet Parsons ein wesentlich breiter aufgestelltes Modell, in dem Psychoanalyse nicht (wie Freud) mit grob vereinfachten Vorstellungen von Gesellschaft verbunden wird, sondern beides gewissermaßen auf Augenhöhe und hinreichend differenziert verbunden werden kann.

Das zeigt auch das Beispiel Krankheit/Gesundheit. Parsons Überlegungen tragen zur Klärung des Kontextes bei, in dem Krankheit wie Therapie operieren. Sie öffnen zudem die Perspektive auf kulturelle Differenzen sowohl im Profil von Therapie selbst als auch in Bezug auf die Rollenbilder, die zumindest im Hintergrund aktiv sind. Dies sind Aspekte, die für Therapieforschung wichtig sind. - Parsons gehört zu den wenigen Soziologen, die systematische Überlegungen zur konzeptionellen Verbindung beider Erkenntnisweisen vorgelegt haben. Es gelingt ihm dabei, ihre Autonomie zu respektieren und sie dennoch sinnvoll aufeinander zu beziehen. Und dies nicht primär zum Zweck der Gesellschaftskritik (die vor allem bei Vertretern der Kritischen Theorie stets Ziel bleibt), sondern im Dienst einer differenzierten Gesellschaftstheorie. Die Leitfrage ist daher bei ihm: Wie ist Gesellschaft möglich? Eine der besonderen Vorteile seines Ansatzes ist dabei, dass er diese Frage unter Einbeziehung psychodynamischer Aspekte in soziologische Modelle beantwortet und so das theoretische Repertoire erweitert.

Parsons ist - teils zu Recht, teils zu Unrecht - für seinen Versuch der Vermittlung von Soziologie und Psychoanalyse kritisiert worden (vgl. dazu Nolte 1970). Vor allem für die Vertreter einer Kritischen Theorie blieb Parsons Ideologe einer „Anpassungslehre“. Tatsächlich hat er Anlass für eine solche Einschätzung geboten. Diese Kritik übersieht jedoch Parsons ' Leistung. Aus heutiger Sicht ist naturgemäß manches patiniert; vieles wäre zu ändern. Aber Parsons hat ein differenziertes Programm der Integration psychoanalytischer Erkenntnisse in soziologische Theorie vorgelegt. Dieses Programm ist nach wie vor aktuell und die Möglichkeiten seines Ansatzes sind noch lange nicht erschöpft.

9 So lassen sich deutliche Parallelen zu Alfred Lorenzers entwicklungstheoretischer Begründung der Psychoanalyse (Lorenzer 1972) erkennen - beide betonen die Bedeutung von praktischer Interaktion als Medium der Vermittlung von Psyche und Gesellschaft. 


\section{Literatur}

Dubin, Robert. 1960. Parsons' Actor: Continuities in Social Theory. American Sociological Review 25: 457-466.

Freud, Sigmund. 1923. Das Ich und das Es. In: Sigmund Freud, Gesammelte Werke Bd. XIII, Frankfurt: Fischer 1999.

Lorenzer, Alfred. 1972. Zur Begründung einer materialistischen Sozialisationstheorie. Frankfurt: Suhrkamp.

Nolte, Helmut. 1970. Psychoanalyse und Soziologie. Die Systemtheorien Sigmund Freuds und Talcott Parsons'. Bern/Stuttgart/Wien: Huber.

Parsons, Talcott. 1937. The Structure of Social Action. New York: Free Press.

Parsons, Talcott. 1950. Psychoanalysis and the social structure. Psychoanalytic Quarterly 19: 371-384.

Parsons, Talcott. 1953. Psychoanalysis and Social Science with special Reference to the Oedipus Problem. In: Twenty years of psychoanalysis, hrsg. Franz Alexander and Helen Ross, New York: W. W. Norton and Company.

Parsons, Talcott. 2002. [1958a]. Freuds Beitrag zur Integration von Psychologie und Soziologie. In: Sozialstruktur und Persönlichkeit, Talcott Parsons, 99-139. Eichborn: Klotz.

Parsons, Talcott. 2002. [1958b]. Definition von Gesundheit und Krankheit im Lichte der amerikanischen Werte und der Sozialstruktur Amerikas. In: Sozialstruktur und Persönlichkeit, Talcott Parsons, 353366. Eichborn: Klotz.

Parsons, Talcott. 1964. The Point of View of the Author. In: The Social Theories of Talcott Parsons. A critical Examination, edt. Max Black, 311-36. Englewood Cliffs: Prentice Hall.

Parsons, Talcott. 1972. Das System moderner Gesellschaften, München: Juventa.

Parsons, Talcott et al. 1953. Working papers in the theory of action. Glencoe, Ill: Free Press.

Parsons, Talcott et al. 1956. Family, Socialization and Interaction Process. London: Routledge \& Kegan Paul.

Johann August Schülein, Dr. phil., Studium der Soziologie, Psychoanalyse und Philosophie in Frankfurt und Gießen, em. o. Univ.-Prof. für Soziologie an der Wirtschaftsuniversität; neuste Publikation zum Thema: Soziologie und Psychoanalyse. Perspektiven einer sozialwissenschaftlichen Subjekttheorie, Wiesbaden: Springer VS 2016. 